Educación Física y Ciencia, vol. 19, n 2, e032, diciembre 2017. ISSN 2314-2561

Universidad Nacional de La Plata.

Facultad de Humanidades y Ciencias de la Educación.

Departamento de Educación Física

\title{
Análisis crítico de la Ley de Henneman
}

\author{
Critical review of Henneman's Law
}

\author{
Andrés Santiago Parodi Feye * \\ * Instituto Superior de Educación Física (ISEF), Universidad de la República, Uruguay | \\ andresparodi2005@yahoo.com
}

\section{PALABRAS CLAVE RESUMEN}

Ley de Henneman

Principio del Tamaño

Unidad motora

\section{KEYWORDS}

Henneman's Law

Size Principle

Motor unit
La Ley de Henneman (también denominada "principio del tamaño") establece que, dado que el tamaño de las motoneuronas determina su umbral de excitación, durante la aplicación de una fuerza muscular las unidades motoras de menor tamaño serán las primeras en reclutarse, y en la medida en que el esfuerzo lo requiera, se irán reclutando más unidades motoras en un patrón estereotipado según su progresivo tamaño. No obstante, durante las actividades propias de la vida diaria o el deporte, este principio adolece de numerosas excepciones. El propósito de este trabajo de revisión es describir, en concordancia con la evidencia científica, en qué condiciones esta Ley no se cumple, o bien se ve significativamente alterada.

\section{ABSTRACT}

Henneman's Law (also known as "size principle") states that, considering that motor neuron's size determines its excitation threshold, during the application of a muscular force the smaller motor units are the first to be recruited, and if the effort is stronger, the other motor units are going to be recruited in a stereotyped way, according to their progressive size. However, during daily living or sport activities, this principle has a number of exemptions. The purpose of this review is to describe, according to the scientific evidence, the conditions under which this principle does not apply or is significantly affected. 


\section{Introducción}

Se conoce como "unidad motora" al conjunto conformado por una motoneurona y el grupo de fibras que inerva, siendo ésta la mínima parte de un músculo que puede hacerse contraer en forma independiente (West, 1991). Representa por consiguiente la unidad funcional básica a partir de la cual el sistema nervioso controla el movimiento, un concepto propuesto por Charles Sherrington en 1925 (Kandel, Schwartz, Jessell, Siegelbaum, \& Hudspeth, 2013).

Cada músculo individual está compuesto por numerosas unidades motoras, pudiendo variar desde unas pocas hasta varios cientos, dependiendo del músculo en cuestión (Mendell, 2005).

En un breve reporte publicado en la revista Science en el año 1957, Elwood Henneman plantea que, a pesar de ser un hecho establecido que el voltaje necesario para excitar eléctricamente los axones es mayor cuanto menor es el diámetro del mismo, en estudios de reflejos espinales las motoneuronas de menor tamaño parecen ser disparadas sinápticamente más fácilmente que las motoneuronas más grandes.

A partir de estos resultados, plantea que las motoneuronas pueden ser graduadas según la facilidad con la cual pueden ser disparadas sinápticamente, siendo las neuronas más grandes las que requieren un estímulo más intenso; en el otro extremo estarían las pequeñas motoneuronas gamma, tan susceptibles a la excitación que incluso la actividad espontánea de la médula espinal es suficiente para mantenerlas continuamente disparando potenciales de acción.

En 1965, Henneman y colaboradores publican cinco artículos científicos en la revista Journal of Neurophysiology, donde se propone la existencia de un principio que denominan "principio del tamaño" (size principle) basado en la correlación muy significativa que existe entre el umbral de descarga o excitabilidad de las motoneuronas y el tamaño de los impulsos que se registran desde sus axones. Según este principio, el tamaño de las motoneuronas determina su umbral de excitación, y por consiguiente el orden de reclutamiento de las mismas también va a variar según su tamaño, siendo las motoneuronas pequeñas las primeras en reclutarse. Este orden de reclutamiento se mantendría inalterado, independientemente de la fuente de excitación neuronal y de los circuitos neuronales que transmiten la misma a dichas motoneuronas (Somjen, Carpenter \& Henneman, 1965).

Considerando que las fibras musculares de tipo I, más pequeñas en tamaño, usualmente están inervadas por motoneuronas a su vez de menor tamaño, y las fibras musculares tipo IIa y IIx, de mayor tamaño, usualmente son inervadas por motoneuronas más grandes, por extensión el principio del tamaño de Henneman se aplicaría también para la contracción de las fibras musculares esqueléticas (Mendell, 2005). Acorde con esto, en una actividad o gesto deportivo donde se requiera la aplicación de poca fuerza se reclutarían únicamente las fibras tipo I (de bajo umbral excitatorio) del grupo muscular involucrado, mientras que si la tarea motriz requiere la aplicación de una fuerza mayor se reclutarán, además de estas fibras, algunas unidades motoras de tipo IIa, de umbral excitatorio más alto. Únicamente cuando la fuerza requerida es muy alta se reclutarán, sumándose a la contracción de los tipos de fibra muscular anteriormente mencionados, fibras musculares de tipo IIx.

Es aceptado que el orden de reclutamiento de unidades motoras de menor a mayor tamaño correlativo a la menor o mayor fuerza de contracción, resulta en un control más preciso de la aplicación de dicha fuerza y por consiguiente del movimiento resultante, particularmente cuando la fuerza aplicada no es de mucha intensidad. Manteniendo el mismo orden de reclutamiento, el sistema nervioso central minimiza las cargas computacionales a lo largo de un amplio rango de resultados esperados (Henneman, Clamann, Gillies, \& Skinner, 1974).

En sus aspectos generales, la Ley de Henneman es aceptada aún hoy en día. No obstante, han sido descriptas numerosas situaciones en las cuales dicha ley no se cumple, o bien se puede ver alterada en sus aspectos fundamentales. En el presente trabajo, habiéndose revisado la literatura científica, se describen y analizan estas posibles situaciones. 


\section{1- El principio de Henneman no se cumpliría si tomamos en consideración un mismo músculo, tal como fue sugerido por dicho investigador}

En un trabajo publicado por Robert Wyman, Ingrid Waldron y Gwendolyn Wachtel (1974) se plantea que, a pesar de que Henneman, en el resumen de su trabajo, deja entrever que el principio del tamaño se cumpliría para un músculo aislado, la mayoría de los experimentos llevados a cabo por su grupo de investigadores demostraron este principio, en realidad, para un grupo de motoneuronas que se originan de un filamento aislado de la raíz ventral de la médula espinal. Por este motivo, según estos autores no queda claro si el Principio de Henneman se cumpliría para el grupo de motoneuronas que terminan en un único músculo, o por el contrario para el grupo de motoneuronas que se originan en un determinado filamento de la raíz ventral.

Con el propósito de dilucidar esto, aplicaron una variedad de estímulos mecánicos en los miembros traseros de gatos adultos, con el propósito de obtener ráfagas de disparos de potenciales de acción en las unidades motoras de los músculos gastrocnemios, encontrando un porcentaje significativo de excepciones a cualquier regla que especifique un orden estereotipado de reclutamiento de las motoneuronas que inervan un mismo músculo.

Por este motivo, los autores plantean la posibilidad de que la Ley de Henneman podría sostenerse si consideramos motoneuronas de una única raíz ventral, cuyos somas son anatómicamente vecinos (aún cuando inerven diferentes músculos), pero no para motoneuronas anatómicamente distantes en la médula espinal, aún cuando inerven el mismo músculo.

\section{2- Henneman experimentó con preparaciones de músculos aislados, lo cual no necesariamente es extrapolable a un sistema fisiológico complejo}

En un artículo de opinión publicado en la revista Frontiers in Human Neuroscience en el año 2014, Parveen Bawa, Kelvin Jones y Richard Stein realizan un análisis crítico del postulado de la Ley de Henneman, mencionando que la precisión de un reclutamiento gradualmente ordenado según el tamaño, que fuera sugerida a partir de preparados reducidos de animales, podría no ser tan precisa en un sistema complejo y "ruidoso". Adicionalmente, el pool de motoneuronas, anatómicamente definido en los experimentos de Henneman y colaboradores, podría no siempre coincidir con el grupo de motoneuronas que son reclutadas para una determinada tarea motriz.

Se agrega a esto, la dificultad experimental que representa medir, en forma precisa, el tamaño de una unidad motora, en un sistema musculo-esquelético complejo.

Por estos motivos, los autores plantean que, hasta tanto no se mida el tamaño y orden de reclutamiento de unidades motoras en rangos relevantes de fuerza durante un comportamiento ecológicamente válido, no podemos asegurar que la Ley de Henneman, obtenida en condiciones experimentales de laboratorio, pueda extrapolarse a los movimientos de la vida cotidiana.

\section{3- En estudios in vivo se ha determinado un patrón de reclutamiento "selectivo" de acuerdo al tipo de movimiento (velocidad, dirección, entre otros).}

En concordancia con la Ley de Henneman, el patrón de reclutamiento de las unidades motoras seguiría un orden estereotipado de reclutamiento. Sin embargo, como lo mencionan en un artículo Roger Enoka y Douglas Stuart (1984) citando a Denny-Brown (1949), el patrón de descarga de las unidades motoras es dependiente de si el músculo en cuestión está funcionando como un motor primario o como un músculo fijador. En este sentido, se plantean los autores, el orden de reclutamiento podría tan solo ser una característica del comportamiento de las unidades motoras para cumplir una determinada función de ese músculo (por ejemplo, flexión, aducción y/o rotación).

En otro artículo (Ter Haar Romeny, Denier van der Gon \& Gielen, 1982) los autores sostienen que en músculos multifuncionales el orden de reclutamiento de las unidades motoras puede modificarse consistentemente 
cambiando la tarea, y consecuentemente, la dirección de la fuerza aplicada por dichos músculos.

Para probar esto, dichos investigadores estudiaron la activación de las unidades motoras del músculo biceps braquial en humanos, durante diferentes acciones: fuerza isométrica de flexión y extensión del codo, fuerza isométrica de pronación y supinación del antebrazo, y fuerza isométrica de rotación interna y externa del hombro. Los resultados mostraron cambios en el orden de reclutamiento de las unidades motoras, consecuentes a cambios en la dirección de la fuerza aplicada. Por ejemplo, unidades motoras con un umbral alto para la flexión tienen tendencia a mostrar un umbral menor cuando están simultaneamente aplicando fuerza en otra dirección.

Si bien estos hallazgos se dieron en un músculo proximal, otros autores citados en este artículo reportaron los mismos hallazgos para músculos polifuncionales del dedo pulgar.

A partir de esto, los autores plantean un patrón de reclutamiento "selectivo" de las unidades motoras, dependiendo de la tarea específica que se está ejecutando, en contraposición al reclutamiento estereotipado hipotetizado en la Ley de Henneman. Sostienen, no obstante, que dicha ley podría aún sostenerse, pero debido a la inervación no homogénea del músculo será necesario especificar en forma exacta la tarea que se está ejecutando.

\section{4- El principio de Henneman no se cumple durante la contracción muscular excéntrica}

La evidencia científica da cuenta de que, durante una contracción muscular excéntrica, se verifica un reclutamiento selectivo de las unidades motoras Ft (o "rápidas"), contradiciendo de esta forma lo que se esperaría encontrar en concordancia con la Ley de Henneman.

En su libro "Eccentric Exercise: Physiology and aplication in sport and rehabilitation", Hans Hoppeler (2014) menciona que la electromiografía de superficie no es capaz, en principio, de proveer la información necesaria para identificar la actividad de una unidad motora aislada, y por tal motivo analiza el trabajo realizado por Nardone y colaboradores (1989), quienes utilizaron para su trabajo electrodos intramusculares.

En dicho trabajo, se estudió la actividad eléctrica de la región del sóleo del músculo triceps sural (región monoarticular, con aproximadamente $70 \%$ de sus fibras de tipo I) y de la región de los grastrocnemios (cabezas biarticulares, con menos de $50 \%$ de sus fibras de tipo I), tanto en contraccion de tipo concéntrica como excéntrica. Se verificó que $15 \%$ de las unidades motoras del sóleo y 50\% de las unidades motoras de los gastrocnemios estaban activas única y exclusivamente durante la contracción de tipo excéntrica, lo que soporta la idea de activación selectiva de unidades motoras de umbral alto, durante este tipo de contracción.

Hoppeler plantea que el mecanismo fisiológico que subyace al reclutamiento selectivo de unidades motoras podría involucrar las células de Renshaw. Este tipo de neuronas inhibitorias, que regulan el patrón de disparo de las motoneuronas, son más fácilmente excitadas por colaterales de motoneuronas de gran tamaño que por motoneuronas de pequeño tamaño, y por consiguiente durante la estimulación de las primeras la acción inhibitoria de estas células sería predominante sobre las segundas, ofreciendo una posible explicación a la inversión de la Ley de Henneman verificada en contracciones de tipo excéntricas.

\section{5- El principio de Henneman no se cumple durante movimientos rápidos o balísticos}

En un artículo de opinión, Bawa, Jones y Stein (2014) sostienen que, en la experiencia de los autores, la situación en la cual el sentido común favorece el reclutamiento selectivo (en contraposición al reclutamiento estereotipado) es durante movimientos ejecutados con máxima velocidad durante un período muy corto de tiempo, es decir, durante un movimiento balístico. La razón que esgrimen, es que en este tipo de movimientos las unidades motoras lentas impedirían la ejecución a máxima velocidad, mientras que el reclutamiento exclusivo de unidades motoras rápidas representaría una situación óptima.

Otros autores (Sale, 1992; Grimby, 1981; Hannerz, 1974) han manifestado evidencia a favor de que la Ley de Henneman no se cumple en movimientos de tipo explosivo. "Esto sugiere que el Sistema Nervioso Central tiene 
mecanismos que permiten activar de modo selectivo unidades motoras que inervan las fibras IIb (rápidas), sin que sea necesario activar antes las fibras lentas" (González Badillo \& Gorostiaga, 2002. Pág. 90).

Minagawa y colaboradores (1978), citado por Siff y Verkhoshansky (2000. Pág. 81) menciona que cuando un movimiento conlleva la realización de grandes fuerzas de corta duración y alta velocidad, se reclutarán las fibras musculares rápidas Ft, mientras se produciría cierta inhibición sobre las fibras lentas St. Por otra parte, Bosco y colaboradores (1982) citados por los mismos autores (pág. 81) sostienen que en un gesto donde se aplique una mínima fuerza, pero la velocidad de acortamiento sea muy elevada, se verificaría un reclutamiento selectivo de fibras Ft, y por consiguiente la Ley de Henneman podría verificarse para cargas de trabajo graduales, pero no para este tipo de gestos.

En concordancia con lo mencionado por estos autores, Remiro, Da Silva y García Manso (2014) en su libro "La halterofilia aplicada al deporte" señalan que:

Experimentalmente se ha podido comprobar (en el músculo sóleo de gato) que cuando las velocidades de acortamiento exceden aproximadamente de $125 \mathrm{~mm} / \mathrm{seg}$ de un máximo potencial de $176 \mathrm{~mm} / \mathrm{seg}$, las unidades motrices lentas (St) puede que no contribuyan al proceso de generar tensión muscular, sino que lo hacen las rápidas (Ft)”(pág. 66).

Adicionalmente a lo expresado en el párrafo anterior, D. Cappa (2000), en su libro "Entrenamiento de la potencia muscular" señala lo siguiente:

Cuando un movimiento es muy rápido, las unidades motoras rápidas pueden ser activadas sin que sea necesario activar las unidades motoras lentas (Smith, 1980). Dentro de esta categoría están incluidos todos los gestos del movimiento de carácter balístico-explosivo (saltos, lanzamientos, golpes)” (pág. 21).

\section{6- En muchas condiciones fisiológicas o patológicas el principio de Henneman no se cumple lo se ve alterado}

El patrón de reclutamiento estereotipado según el tamaño de la unidad motora, fue originalmente descripto para movimientos ejecutados por organismos sanos. No obstante, en situaciones tales como el envejecimiento, reinervación, dolor o fatiga, este patrón puede verse alterado (Bawa, 2014).

Con el envejecimiento, ocurre la muerte de motoneuronas que dejan huérfanas sus correspondientes fibras musculares. La reinervación por parte de otra motoneurona vecina puede derivar en una modificación del tamaño de la unidad motora, y bajo estas circunstancias el orden de reclutamiento podría verse afectado, con una pérdida en la precisión del control motor (Gordon, Thomas, Munson, \& Stein, 2004).

Por otra parte, se ha establecido en sujetos sanos, que durante los procesos de fatiga muscular se verifica un patrón de activación rotatorio entre las unidades motoras (Manning, Miller, Burnham et al, 2010). Si la rotación ocurre entre unidades motoras de tamaño muy similar, la precisión del gesto motor podría no verse afectada; sin embargo, si las unidades motoras fueran de tamaños muy diferentes, esto incrementaría el ruido en el resultado motriz (Bawa, 2014).

Otra situación en la cual el principio de Henneman se podría ver afectado, es durante la contracción muscular durante procesos de dolor. A este respecto, Tucker y colaboradores (2009) sostienen que las estrategias de reclutamiento de unidades motoras en estas condiciones se ven alteradas, con el propósito de mantener los niveles de fuerza.

En su trabajo, los autores concluyen que la menor frecuencia de descarga de las unidades motoras que se verifica durante procesos dolorosos, es acompañado por cambios en la población de unidades motoras utilizadas para el mantenimiento de la fuerza producida. De esta forma, el patrón de reclutamiento previsto por la Ley de Henneman se vería alterado. 


\section{7- El principio de Henneman no se verifica en músculos respiratorios primarios durante la respiración voluntaria e involuntaria}

Ha sido evidenciado que, en algunas condiciones, la Ley de Henneman no se verifica en músculos respiratorios primarios, tanto durante la respiración voluntaria como involuntaria.

Para el primer caso, en un estudio (Butler, McKenzie, \& Gandevia, 1999) se evidenció grandes alteraciones en el orden de reclutamiento de las unidades motoras del diafragma, el cual se mostraba dependiente de la tarea respiratoria asignada a los sujetos experimentales. En este mismo trabajo se mencionan reportes de cambios en el patrón de reclutamiento para los músculos intercostales paraesternales, durante el sueño y la hiperventilación voluntaria.

\section{8- El principio de Henneman no se cumple durante la electroestimulación}

La electroestimulación transcutánea o percutánea de un músuclo o grupo muscular, es una estrategia bien fundamentada y comunmente utilizada por kinesiólogos y entrenadores deportivos, con el propósito de incrementar la fuerza muscular de pacientes debilitados por una determinada patología, o bien para potenciar los efectos logrados por los deportistas a través del entrenamiento de fuerza "tradicional".

En el trabajo publicado por Delitto y Snyder-Mackler (1990), se parte de la base de que hay una importante diferencia, bien documentada, entre la contracción muscular acaecida por un estímulo nervioso voluntario, y aquella que se produce a partir de la electroestimulación externa. En este sentido plantean que, considerando que la corriente tiende a tomar el camino de menor resistencia eléctrica, cuando se aplica un estímulo eléctrico exógeno se reclutan más fibras nerviosas de mayor tamaño (y menor resistencia eléctrica) que fibras nerviosas de menor tamaño (y mayor resistencia eléctrica).

Los autores agregan que, a pesar de que este patrón de reclutamiento varía en cierta medida con la geometría del nervio y el lugar de aplicación de los electrodos, en términos generales se puede afirmar que en este caso se daría una activación opuesta a lo que plantea la Ley de Henneman.

En concordancia con esto, en otro trabajo de investigación (Trimble y Enoka, 1991) se concluye que la aplicación de estimulación eléctrica percutánea en los miembros inferiores de humanos, puede alterar el patrón de reclutamiento de las unidades motoras y la población particular de unidades motoras activadas por un determinado estímulo, en comparación con lo que acontece durante una contracción de tipo voluntaria.

Otros trabajos anteriores (Garnett \& Stephens, 1981) ya habían dado cuenta de que el input aferente de la estimulación transcutánea resulta en un input inhibitorio de las motoneuronas alfa que inervan las fibras musculares de tipo I, y en un input exitatorio de las motoneuronas alfa que inervan las fibras musculares de tipo II a nivel de la médula espinal, resultando en un patrón de reclutamiento selectivo, diferente al planteado por el principio del tamaño.

En lo que respecta a la respiración involuntaria, el trabajo de revisión de Jane Butler (2007) afirma que, en las respiraciones involuntarias se han evidenciado alteraciones en el patrón de reclutamiento de unidades motoras del músculo diafragma, en un rango similar al observado en tareas respiratorias voluntarias, algo que indicaría una violación al principio del reclutamiento estereotipado sugerido por Henneman.

A modo de conclusión: sería absolutamente inadecuado afirmar que la Ley del tamaño descripta por Henneman no es correcta, o que carece de validez. No obstante, y a partir de las evidencias mencionados ut supra, es posible argumentar que dicha ley se cumple solo en determinadas situaciones particulares, y consecuentemente no es adecuada su extrapolación a todos los gestos motrices de la vida cotidiana o deportiva. 


\section{Bibliografía}

Bawa, P., Jones, K., \& Stein, R. (2014). Assessment of size ordered recruitment. Frontiers in Neuroscience , 1-3.

Butler, J. (2007). Drive to the human respiratory muscles. Respiratory Physiology \& Neurobiology, 159 (2) 115126.

Butler, J., McKenzie, D., \& Gandevia, S. (1999). Discharge properties and recruitment of human diaphragmatic motor units during voluntary inspiratory tasks. Journal of Physiology , 518(3) 907-920.

Delitto, A., \& Snyder-Mackler, L. (1990). Two Theories of Muscle Strength Augmentation Using Percutaneous Electrical Stimulation . Physical Therapy, 70(3) 158-164.

Enoka, R., \& Stuart, D. (1984). Henneman's Size Principle: Current Issues. Trends in Neuroscience , 7(7) 226-228.

Garnett, R., \& Stephens, J. (1981). Changes in the recruitment threshold of motor units produced by cutaneous stimulation in man. . Journal of Physiology , 311(1) 463-473.

González Badillo, J., \& Gorostiaga, E. (2002). Fundamentos del entrenamiento de la fuerza. Barcelona: INDE.

Gordon, T., Thomas, C., Munson, J., \& Stein, R. (2004). The resilience of the size principle e in the organization of motor unit properties in normal and reinnervated adult skeletal muscles. Canadian Journal of Physiology and Pharmacology , 82 (8-9), 645-661.

Grimby, L., Hannerz, J., \& Hedman, B. (1981). The fatige and voluntary discharge properties of single motor units in man. Journal of Physiology , 316(1), 545-554.

Henneman, E., Clamann, H. P., Gillies, J. D., \& Skinner, R. (1974). Rank order of motoneurons within a pool: law of combination. Journal of Neurophysiology , 37(6), 1338-1349.

Kandel, E., Schwartz, J., Jessell, T., Siegelbaum, S., \& Hudspeth, A. (2013). Principles of Neural Science (5a ed.). New York: McGraw Hill.

Manning, C., Miller, T., Burnham, M., Murnaghan, C., Calancie, B., \& Bawa, P. (2010). Recovery of human motoneurons during rotation. Experimental Brain Research , 204(1), 139-144.

Mendell, L. (2005). The size principle: a rule describing the recruitment of motoneurons. Journal of Neurophysiology , 93(6), 3024-3026.

Remiro, G., Da Silva, M., \& García Manso, J. (2014). La halterofilia aplicada al deporte. Wanceulen.

Siff, M., \& Verkhoshansky, Y. (2000). Superentrenamiento. Paidotribo.

Somjen, G. C., Carpenter D. O. \& Henneman E. (1965). Responses of motoneurons of different sizes to graded stimulation of supraspinal centers of the brain. Journal of Neurophysiology , 28(5), 958-965.

ter Haar Romeny, B. M., Denier van der Gon, J. J.\& Gielen, C.C. (1982). Changes in recruitment order of motor units in the human biceps muscle. Experimental neurology. Nov; 78(2), 360-368.

Trimble, M., \& Enoka, R. (1991). Mechanisms underlying the training effects associated with neuromuscular electrical stimulation . Physical Therapy, 71(4), 273-280.

Tucker, K., Butler, J., Graven-Nielsen, T., Riek, S., \& Hodges, P. (2009). Motor Unit Recruitment Strategies Are Altered during Deep-Tissue Pain. The Journal of Neuroscience , 29(35), 10820-10826.

West, J. (1991). Physiological basis of medical practice (12a ed.). Baltimore: Williams \& Wilkins.

Wyman, R., Waldron, I., \& Watchel, M. (1974). Lack of Fixed Order of Recruitment in Cat Motoneuron. Experimental Brain Research , 20(2), 101-114. 